\title{
FLAT AND PROJECTIVE CHARACTER MODULES
}

\author{
THOMAS J. CHEATHAM AND DAVID R. STONE
}

\begin{abstract}
ABSTRACr. We characterize left coherent, left Noetherian, and left Artinian rings by the flatness, projectivity, and injectivity of the character module of certain left $\boldsymbol{R}$-modules.
\end{abstract}

Introduction. The character module of a left (right) $R$-module $M$ is the right (left) $R$-module $M^{+}=\operatorname{Hom}_{Z}(M, Q / Z)$ where $Z$ denotes the group of integers and $Q$ denotes the additive group of rational numbers. Character modules have played an important role in the study of rings through their modules. See, for example, [3]-[6], [8], and [9]. Lambek [6] proved that, over any ring, a module is flat if and only if its character module is injective (equivalently absolutely pure). It is easy to show that a left perfect ring is characterized by the property: A module is projective if and only if its character module is injective. We shall consider the dual conditions and determine those classes of rings for which a module has one of the properties absolutely pure or injective if and only if its character module has one of the dual properties flat or projective. The main result of this paper is a characterization of a left Artinian ring as a ring over which a left $R$-module is injective if and only if its character module is projective. This removes an unnecessary hypothesis from Ramamurthi [8, Proposition 6, p. 182] and proves the converse.

Results. Let $R$ denote an associative ring with identity. Following Ramamurthi [8] we call an $R$-module $M$ a PC-module (FC-module) if $M^{+}$is a projective (respectively flat) $R$-module on the opposite side. A module is absolutely pure if it is a pure submodule of every over-module. The following lemma will be useful.

Lemma 1. Let I be any index set. Let $\left(A_{i}\right)_{i \in I}$ be any family of left (or right) $R$-modules. Then

(1) $\bigoplus A_{i}$ is a pure submodule of $\Pi A_{i}$.

(2) If for each $i \in I, B_{i}$ is a pure submodule of $A_{i}$, then $\Pi B_{i}$ is a pure submodule of ПI $\boldsymbol{A}_{\boldsymbol{i}}$.

The proof of Lemma 1 is straightforward. We note that if $M$ is an FC-module then $M$ is absolutely pure. This is an easy consequence of the fact that every character module is pure-injective. We begin by studying the weakest of the four conditions mentioned in the introduction. A result of Würfel [9] is included for completeness.

Received by the editors April 1, 1980.

1980 Mathematics Subject Classification. Primary 16A50, 16A52.

Key words and phrases. Character module, flat, projective, injective, coherent, Noetherian, Artinian. 
THEOREM 1. The following statements are equivalent:

(1) $R$ is a left coherent ring.

(2) ${ }_{R} M$ is absolutely pure if and only if $M$ is an FC-module.

(3) ${ }_{R} M$ is absolutely pure if and only if $M^{++}$is an injective left $R$-module.

(4) $M_{R}$ is flat if and only if $M^{++}$is a flat right $R$-module.

Proof. (1) $\leftrightarrow$ (2) follows from Würfel [9, Satz 1.6, p. 383] and the previous note.

$(2) \leftrightarrow(3)$ is a consequence of the well-known result of Lambek [6].

(3) $\leftrightarrow$ (4) Let $M$ be a flat right $R$-module. Then $M^{+}$is injective so $M^{+++}$is injective and hence $M^{++}$is flat. Conversely if $M^{++}$is flat then $M$, being a pure submodule of $M^{++}$(see [9, Exercise 41, p. 48]), is flat, too.

(4) $\leftrightarrow$ (1) Let $\left(M_{i}\right)_{i \in I}$ be a family of flat right $R$-modules. We show that $\Pi M_{i}$, $i \in I$, is flat. It will follow that $R$ is left coherent. The index set in the following statements is $I$. $\bigoplus M_{i}$ is flat so $\left(\bigoplus M_{i}\right)^{++} \cong\left(\Pi M_{i}^{+}\right)^{+}$is flat by (4). But $\bigoplus M_{i}^{+}$ is a pure submodule of $\Pi M_{i}^{+}$so $\left(\Pi M_{i}^{+}\right)^{+} \rightarrow\left(\bigoplus M_{i}^{+}\right)^{+} \rightarrow 0$ splits. Thus, $\Pi M_{i}^{++}$ $\cong\left(\bigoplus M_{i}^{+}\right)^{+}$is flat. Since $\Pi M_{i}$ is a pure submodule of $\Pi M_{i}^{++}, \Pi M_{i}$ is flat.

Now we strengthen the property on the module and consider the condition: $A$ module is injective if and only if its character module is flat.

THEOREM 2. The following statements are equivalent:

(1) $R$ is left Noetherian.

(2) ${ }_{R} M$ is injective if and only if ${ }_{R} M^{++}$is injective.

(3) ${ }_{R} M$ is injective if and only if ${ }_{R} M$ is an FC-module.

Proof. (2) $\leftrightarrow(3)$ is obvious.

(1) $\rightarrow$ (2) If ${ }_{R} M^{++}$is injective, then ${ }_{R} M$ is absolutely pure. Since $R$ is left Noetherian, ${ }_{R} M$ is injective (Megibben [7, Theorem 3]). If ${ }_{R} M$ is injective then by Theorem 1(3) ${ }_{R} M^{++}$is injective.

$(2) \rightarrow$ (1) We show that each absolutely pure left $R$-module $M$ is injective. Let ${ }_{R} M$ be absolutely pure. There is a pure exact sequence $(0) \rightarrow M \rightarrow E \rightarrow X \rightarrow(0)$ where $E$ is injective. Thus (0) $\rightarrow X^{+} \rightarrow E^{+} \rightarrow M^{+} \rightarrow(0)$ splits and so does (0) $\rightarrow$ $M^{++} \rightarrow E^{++} \rightarrow X^{++} \rightarrow(0)$. Then $M^{++}$is injective since $E^{++}$is. By (2) $M$ is injective.

Next, we strengthen the requirement on the character module and consider: A module is absolutely pure if and only if its character module is projective.

THEOREM 3. The following statements are equivalent:

(1) A left $R$-module is absolutely pure if and only if it is a PC-module.

(2) $R$ is left coherent and right perfect.

Proof. (1) $\rightarrow$ (2). From Würfel [9, Satz 1.6, p. 383] it follows that $R$ is left coherent. To prove $R$ is right perfect we use Chase [2, Theorem 3.1] for right $R$-modules. Let $J$ be an infinite set whose cardinality is greater than the cardinality of $R$. For each $j \in J$, let $R_{j}$ denote a copy of the right $R$-module $R$. All sums and products will be taken over the index set $J$. Since $\bigoplus R_{j}$ is flat, $\left(\bigoplus R_{j}\right)^{+} \simeq \Pi R_{j}^{+}$is an injective left $R$-module. By Lemma $1 \oplus R_{j}^{+}$is a pure submodule of $\Pi R_{j}^{+}$, so by assumption $\left(\bigoplus{R_{j}}^{+}\right)^{+} \cong \Pi{R_{j}}^{++}$is a projective right $R$-module. But $\Pi R_{j}$ is a 
pure submodule of $\Pi R_{j}^{++}$and hence a pure submodule of a free right $R$-module. According to Chase [2, Theorem 3.1] $R$ is right perfect.

(2) $\rightarrow$ (1) Since $R$ is left coherent a left $R$-module is absolutely pure if and only if it is an FC-module. Since $R$ is right perfect a right $R$-module is flat if and only if it is projective. (1) follows easily.

Finally we consider the remaining condition: A module is injective if and only if its character module is projective.

THEOREM 4. The following statements are equivalent:

(1) $R$ is left Artinian.

(2) ${ }_{R} M$ is injective if and only if it is a PC-module.

Proof. (1) $\rightarrow$ (2). A left Artinian ring is left Noetherian and perfect on both sides. Over a left Noetherian ring each absolutely pure left $\boldsymbol{R}$-module is injective. Now apply Theorem 3.

$(2) \rightarrow(1)$. We will prove that $R$ is left Noetherian and right perfect (cf. Björk [1]). Let $\left(E_{i}\right)_{i \in I}$ be any family of injective left $R$-modules. All sums and products will be taken over the index set $I$. Since $\bigoplus E_{i}$ is a pure submodule of $\Pi E_{i}$, $\left(\bigoplus E_{i}\right)^{+}$is isomorphic to a direct summand of (II $\left.E_{i}\right)^{+}$. The latter is projective since II $E_{i}$ is injective. Thus $\left(\bigoplus E_{i}\right)^{+}$is projective and by hypothesis $\bigoplus E_{i}$ is injective. This proves $R$ is left Noetherian. An alternate proof could be obtained by proving that each absolutely pure left $R$-module is injective.

Now, let $J$ be an infinite set whose cardinality is greater than the cardinality of $R$. For each $j \in J$, let $R_{j}$ denote a copy of the right $R$-module $R_{R}$. All sums and products are taken over the index set $J$. Now each ${R_{j}}^{+}$is an injective left $R$-module so by (2) $\left(\bigoplus R_{j}^{+}\right)^{+} \cong \Pi R_{j}^{++}$is a projective right $R$-module. But $\Pi R_{j}$ is a pure submodule of $\Pi R_{j}^{++}$and thus a pure submodule of some free right $R$-module. Applying Chase [2, Theorem 3.1, p. 464] we conclude that $R$ is right perfect.

This removes an unnecessary hypothesis of Ramamurthi [8, Proposition 6, p. 182] and proves the converse. We are indebted to Professor Ed Enochs for several stimulating and helpful conversations.

\section{REFERENCES}

1. J. E. Björk, Rings satisfying a minimum condition on principal ideals, J. Reine Angew. Math. 236 (1969), 112-119.

2. S. U. Chase, Direct products of modules, Trans. Amer. Math. Soc. 97 (1960), 457-473.

3. T. Cheatham and J. Smith, Regular and semisimple modules, Pacific J. Math. 65 (1976), 315-323.

4. R. R. Colby, Rings which have flat injectives, J. Algebra 35 (1975), 239-252.

5. E. E. Enochs, $A$ note on semihereditary rings, Canad. Math. Bull. 16 (1973), 439-440.

6. J. Lambek, $A$ module is flat if and only if its character module is injective, Canad. Math. Bull. 7 (1964), 237-243.

7. C. Megibben, Absolutely pure modules, Proc. Amer. Math. Soc. 26 (1970), 561-566.

8. V. S. Ramamurthi, On modules with projective character modules, Math. Japon. 23 (1978), 181-184.

9. Bo Stenström, Rings of quotients, Die Grundlehren der Math. Wissenschaften, Band 217, Springer-Verlag, Berlin and New York, 1975.

10. T. Würfel, Über absolut reine Ringe, J. Reine Angew. Math. 262-263 (1973), 381-391.

Department of Mathematics, SAmford University, Birmingham, Alabama 35209

Department of Mathematics, Georgin Southern College, Statesboro, Georgia 30458 